
\title{
BResarch Soute \\ Classification and Review of Health Financing Frameworks: What do they Focus on to Assess and Improve the Situation?
}

\section{Minoo Alipouri Sakha}

Iran University of Medical Sciences

Mohammad Bazyar. ( $\sim$ bazyar.mohamad@gmail.com )

3 Department of Health Promotion, Faculty of health, Ilam University of Medical Sciences, Ilam, Iran

Arash Rashidian

Department of Science, Information and Dissemination, World Health Organization Regional Office for the Eastern Mediterranean, Cairo, Egypt

\section{Research Article}

Keywords: Health financing assessment, universal health coverage, PubMed database

Posted Date: February 23rd, 2021

DOI: https://doi.org/10.21203/rs.3.rs-223617/v1

License: (9) This work is licensed under a Creative Commons Attribution 4.0 International License. Read Full License 
1 Classification and review of health financing frameworks: what do they

$r \quad$ focus on to assess and improve the situation?

$\varepsilon \quad$ Minoo Alipouri Sakha ${ }^{1}$, Arash Rashidian ${ }^{2}$, Mohammad Bazyar $^{3 *}$

0

1 Department of Health Education \& Promotion, Deputy of Health, Iran University of Medical Sciences, Tehran, Iran

2 Department of Science, Information and Dissemination, World Health Organization Regional Office for the Eastern Mediterranean, Cairo, Egypt

3 Department of Health Promotion, Faculty of health, Ilam University of Medical Sciences, Ilam, Iran

9

*Corresponding author: Mohammad Bazyar. Assistant Professor in Health Policy, Department of Health Promotion, Faculty 
10 Abstract

17 Objective

iv Health financing assessment is of growing interest to policy makers to develop reform in strategies towards achieving universal health coverage. Different frameworks for assessing of 19 health financing systems have been developed. The purpose of this study is to review the $r$. current frameworks for understanding and assessing health financing and draw out the dimensions of conceptual frameworks and how different concepts are used in relation to the concept of health financing assessment. Lessons learned from different frameworks would guide the future use and formulation of such frameworks.

\section{Methods}

We carried out a systematic search strategy, using the PubMed database to find peer reviewed published literature. A search using generic search engines (Google Scholar and the Google) was also performed through a less structured search to find additional documents and reports published. To increase the comprehensiveness of search, Websites of World Health Organization (WHO) and World Bank were also searched. We included any type of report or peer reviewed journal article that reported frameworks to assess, review and map financing functions and sub-functions and highlight different factors that affect it.

\section{Results}

Fifteen frameworks/tools developed during 2001 until 2020 were found. We classified frameworks by grouping them into systems approach -analytical frameworks ,indicatorbased, and descriptive/ narrative categories. These frameworks vary in the concepts, functions and processes, dimensions, strategies and meet various international or countryspecific challenges. 
\&) Conclusion

\&r The process of framework development for health financing review is becoming more \&r comprehensive with pushing universal health coverage to the international agenda. Despite « $\quad$ being diverse, various health financing frameworks/ tools offer synergistic views to the health so financing system and provide a comprehensive picture of the health financing system. While $\leq \tau$ some frameworks covered the majority of the elements, not any of the frameworks on its own $\Sigma \vee \quad$ covered all the elements. Health financing review is a laborious endeavor, which usually ¿ needs diverse data and an expert team. It is sometimes infeasible to access ready and user« friendly information; therefore, it is important to apply them with some flexibility.

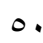


or Health financing occupies a central role in Universal Health Coverage (UHC), one of the ov Sustainable Development Goals (SDGs) health targets to be committed (1). World health o1 report 2000 highlights the functions of a health financing system: the mobilization, ०9 accumulation and allocation of money to cover the health needs for all the people (2). In ฯ. its 2010 World Health Report, the World Health Organization defines the concept of health 7) financing for universal coverage as "financing systems need to be specifically designed to ir provide all people with access to all needed health services of sufficient quality without being ir exposed to financial hardship" (3). Health financing as one of the structural aspects of health role in universal health coverage $(1,4)$.

ir Health policy makers around the world are faced with increasingly difficulties to sustain IV sufficient financing for health care (5). Tens of millions of people the world over either do not $\checkmark$ have access to health care services they need or they have to pay for it, which are catastrophic 79 or impoverishing (3).

$\vee$. Many countries, particularly low and middle-income ones, are developing health financing systems that enable them to sustain or to move towards universal health coverage. However, there is still a gap in evidence and knowledge about national and country-level health systems to achieve improved health outcomes, equity, and public satisfaction, play an essential or impoverishing (3).

financing systems that perform well in terms of improving health outcomes and financial protection $(3,6,7)$.

To date, multiple frameworks to assess or review health financing have been developed. The ultimate goal of a conceptual framework is to provide a "starting point" for a national health reform strategy (8). The purpose of some frameworks is to identify the factors involved in health financing, and suggest policy options." They provide a systematic tool for policy- 
makers to analyze functions and interactions of different dimensions of health systems that must be considered for developing policy alternatives and reforms (9). They help synthesize data

systematically to fill the gap between pure academic research and the needs of policy-makers (10), distributional impact of various sources of health system funds and the logic of actors $(9$, $11,12)$.

Researchers with different perspectives attempted to apply different criteria for review of health financing system. Some authors framed their approach on the two WHO 2000 and 2010 reports while others attempted to explain the relationships between contextual and structural elements of the health financing system. In order to be evidence-based, decisions about financing must consider all functions of health financing and factors that affect this component. We conducted a comprehensive review of the existing published knowledge on health financing assessment. More specifically, we: (1) review the current frameworks for understanding and assessing health financing and (2) draw out the dimensions of conceptual frameworks and how different concepts are used in relation to the concept of health financing assessment. Lessons learned from different frameworks would guide the future use and formulation of proper reforms to improve health financing system performance. We described and compared health financing frameworks to guide policymakers when selecting a framework to use for assessing health financing and help them use evidence for health finance policy making. This is accomplished by giving a brief overview of frameworks focusing on the dimensions and metrics for each one and summarizing qualitatively the key dimensions, which the majority of the included frameworks highlighted for health financing review. 


\section{1.r Method}

1.r We carried out a systematic search strategy, using the PubMed database to find peer reviewed

1. $\{$ published literature up to April 2020. The search was guided by the following search terms:

1.0 health, finance*, framework*, assess*. A search using generic search engines (Google

1.7 Scholar and the Google) was also performed through a less structured search using general

1.V terms representing 'health financing framework' and 'health financing' to find additional

1.1 documents and reports published. To increase the comprehensiveness of search, Websites of

1.9 World Health Organization (WHO) and World Bank were also searched for relevant grey

11. literature and the reference lists of all selected documents were scanned. The search results

111 were narrowed down using three criteria: English-language literature, review articles and

IIr timeframe since 2000 .

IIr Our initial intention was to focus on reviews, and meanwhile on the usages of framework(s)

II $\leq$ in assessing health financing systems. Therefore, the final selection of papers included a

110 combination of both papers, which either have proposed a framework or used a conceptual

117 framework to frame the design of their research project or to explain the outcomes of policy

IIV assessment of how a country's health financing are organized.

$11 \wedge$ We included any type of report or peer reviewed journal article that reported frameworks to

119 assess, review and map financing functions and sub-functions and highlight different factors

1r. that affect it, in order to identify all functional and non-functional dimensions needed to be

Ir) considered in assessing or reviewing of health financing system. Towards this end, analytical,

ir descriptive and even prescriptive frameworks were selected. The frameworks that analyze the

irr performance of the entire health system are also considered since health financing is a main

Ir $\varepsilon \quad$ component of health system. 
irv Fifteen frameworks/tools developed during 2001 until 2020 were found. The identified

ir frameworks or tools goals were "assessing" or "reviewing" health financing. We classified

i $\mathrm{f}$ frameworks by grouping them into systems approach -analytical frameworks ,indicator-

ir. based, and descriptive/ narrative categories. These frameworks vary in the concepts,

ir) functions and processes, dimensions, strategies and meet various international or country-

irr specific challenges.

Table 1: An illustrative list of health financing conceptual frameworks

Systems approach -Analytical frameworks

Kutzin suggested an analytical tool for critical assessing of the functions of health financing. The framework identifies three pillars (1) "explicit health financing policy objectives as I乏. assessment criteria"; (2) "a function-based framework as a basis for describing health Iミ) financing systems"; and (3) "analysis of fiscal context". Kutzin conceptualized the organizational and institutional arrangements of components of health financing and categorized options under each function. He also stressed on the information and implementation of regulation as key policy tools to enhance the insurance function $(7,13,14)$.

The framework developed by William Hsiao lists the causal components which explain the system's outcomes, health status, financial risk protection and consumer satisfaction as final goals; access, quality and efficiency of service delivery as intermediate outcomes; and financing, organizational structure, payment mechanisms, regulation and information as means (15).

10. Bertone and Meessen proposed a framework drawing from concepts of New Institutional 101 Economics (NIE). It aims to provide a better understanding of how institutional arrangements 
shape the performance of health systems. It built upon the idea of the institutional design and organizational practice for health financing performance proposed by Mathauer and Carrin. This framework consists of seven dimensions: 'institutional arrangements', 'enforcement mechanisms', 'property rights', 'incentives', 'intrinsic and extrinsic motivation', 'behavioral changes' and 'organization's performance'. Meessen highlighted the relations between these dimensions and stated that power and motivations of the key actors, enforcement mechanisms and the content of rules shaped the 'property rights'. These decision rights analyses include the decision and earning rights of the main actors, which in return defines the alignment of actor's incentives with principals' objectives. Incentives usually take advantage of intrinsic and extrinsic motivation and determine behavioral changes. Assessing the contribution of behaviors to the goals of health system is the last step of analysis (16). The framework should lead to a better understanding of the important role of institutional analysis in the design and implementation of performance-based financing (PBF) interventions.

WHO Health Financing Strategy for the Asia Pacific Region (2010-2015) framework includes "issues and challenges", "main policy objectives", and "actions" to take by member states and WHO. This framework contains some robust strategic areas to analyze health financing situations and identifies specific actions to achieve universal coverage. Eight strategic areas are public spending on health, effective aid for health, efficient rationalizing health expenditures, prepayment and pooling, provider payment methods, safety-net mechanisms for the poor and vulnerable, evidence-based policymaking and monitoring and evaluation (17).

World Bank conceptual framework provides practical steps on "how" to introduce health insurance in low-income countries. To simplify the complicated health insurance issue, this framework has divided health insurance into eight design elements including: Feasibility of Establishment Health Insurance (gap analysis, political support, political and financial capacity, provider capacity and socioeconomic factors); Financing Mechanisms (different kinds of health insurance); Population Coverage (identifying different groups of population to 
i $\vee$ be covered especially hard-to-reach populations such as low-income, rural, informal sector workers); Benefits Package; Provider Engagement; Organizational Structure; Operational processes; Monitoring and Evaluation of Health Insurance Schemes. The purpose of this framework is to help health insurance designers from middle and low-income countries with step-by-step activities to scale up existing health insurance funds or to design a health insurance plan from the beginning. For each design element, the framework helps policy makers to identify the political, economic, social, and institutional opportunities and barriers, and alternative approaches so they can adjust them according to the real conditions in their countries in a way to move health insurance forward on a rational and feasible path (18).

In the study by Bazyar et al., the above framework was applied to analyze challenges ahead of merging health insurance funds in Iran as a country with fragmentation in health risk pools to create a single national fund. In their study, they introduced a new framework, added three new aspects to the Work Bank framework, and extended it to 11 elements. Three new dimensions were "Justification of the consolidation process; the explicit definition of the policy objectives", "Stewardship", and "Health Service Delivery". Stewardship refers to alignment of health insurance system with the policies of the broader health care system especially in countries with a purchaser-provider split. "Health service delivery" refers to how health insurance schemes provide health care services for their beneficiaries and differences between them. This item also refers to the effect of consolidation on the quality and quantity of heath care services delivered by the health care providers (19).

Resource Tracking and Management" (RTM) framework provides a comprehensive analysis of health financing by bringing 5 key resource tracking efforts including Resource Mobilization, Resource Allocation, Resource Utilization, Resource Productivity and Resource Targeting into one comprehensive structure. It collects information by different tools such as fiscal space analysis; Public Expenditure Reviews (PERs), Cost-effectiveness analysis and priority 
$r \cdot r \quad$ setting, planning/budget process, and National Health Accounts (NHAs), Public Expenditure

r.£ Tracking Surveys (PETs), Quantitative Service Delivery Survey (QSDS) or facility surveys,

r.o and Benefit Incidence Analysis (BIA) (20).

$r \cdot \uparrow$ The aim of the accounting framework (SHA) is to provide a clear and transparent picture of a

$r \cdot v$ country's financing schemes, institutional units and key transactions (flows). The SHA

$r \cdot \wedge$ framework makes it possible to analyze different issues as follows: the revenue- collection of a

r. 9 particular financing scheme; mobilizing of revenues of particular institutional units of the

r. economy; the share of the main financing schemes in a country's health care sector; the

Y) (purchased services under the different financing schemes; resources allocation of the different

IIT financing schemes among the different services; financing of a particular health care service;

ir allocation of the resources of the different financing schemes among the different groups of

Y I $\leq$ beneficiaries; flow of money; management of health care financing; and institutional

r. arrangements of governing the funds of financing schemes (21).

r19

Y YIndicator-based frameworks

r) U.S. Agency for International Development designed a structured and indicator-based Y19 approach to provide a rapid and comprehensive assessment of a country's health system. In rr. Health Systems Assessment Approach (HS20/20), data related to health, economics and rr demographics indices are retrieved by desk review of relevant documents and structured rr stakeholder interviews. Health financing strengths and weaknesses are diagnosed by data rr analysis and then based on country priorities and gaps, recommendations and policies will be rYs developed (22).

rro OASIS framework proposed by WHO uses secondary data, document review, and rry stakeholder interviews. It consists of three steps: a health financing performance assessment, a 
rrv more detailed institutional-organizational analysis and identification of options and necessary rrA changes required for health financing improvements. This framework identifies six types of rrq bottlenecks in the institutional design and organizational practice, and six entry points of $r$ r. proposed changes to identify as, enhancing health financing performance, which focuses on rr modifying legal and regulatory provisions, improving organizational practice through rrs strengthening organizational capacity and enforcement practices. It applies to review either the rr whole health financing system or specific health financing schemes (11).

r $\{$ The Public Financial Management Performance - Self-Assessment (PFMP-SA) framework is rro derived from the PEFA framework, adapted to the needs of the health sector, which promote rrı ministries of health to conduct self-assessments of the performance of their PFM systems. The rmv PFMP-SA include a range of 12 modified 'standard' PFM indicators as follows: "1. ґґ Expenditure against original budgets links to programs, priorities, and results 2. Extent and rrq monitoring of expenditure payment arrears toward suppliers, contractors, and employees 3. r£. stakeholders' access to key fiscal information 4. Ministry's practices and success in rs elaborating medium-term planning, budgeting and expenditure frameworks 5. Cash flow $r \leqslant$ predictability and control over timely budget execution 6. Access and control over payroll by r $\leqslant$ MOH 7. Adequate, efficient, and transparent procurement system 8. Formal and documented $r \leqslant \varepsilon$ internal controls 9. Internal audit functions 10. The accounting system provides coherent r $\_$information on resources reaching lower levels of service delivery 11 . The accounting system $r \leq 4$ can produce timely periodic reports 12 . The $\mathrm{MOH}$ is receiving and using financial information $Y \leqslant \vee$ provided by donors for budgeting and reporting on project and program aid." The assessment $r \leqslant \wedge$ evaluates the indicators impact on the six core dimensions: credibility of the budget; ५ १ comprehensiveness and transparency; policy-based budgeting; predictability and control in ro. budget execution; accounting, recording, and reporting; and external scrutiny and audit (23). 
ror Kutzin and McIntyre Developed "Guidance on Conducting a Situation Analysis of Health ror Financing for Universal Health Coverage "which can be used as a complement to OASIS or ros independently. It is a tool aims to assess a country's health financing system relative to the roo goal of universal health coverage (UHC) and the challenges faced in moving towards UHC. ror The approach is predominantly qualitative with some supporting quantitative data. It is a rov comprehensive guidance for situation analysis, and unlike other frameworks; this model goes ros beyond simple instructions and can be used by users to assess performance in practice. The ro9 authors argued key contextual factors including health expenditure patterns and implications, чฯ. public sector financial management, and the structure of public administration and fiscal

rT)

rTr

rฯT

TYE

rro

rTT

rTV

rTA

r79

rV.

rVI

TVY

TVT

TV

rVo

rVT context are essential for a full analysis of health financing arrangements. They also highlighted relative power of different actors in the budget decision-making process, expenditure control measures, extent of decision-making autonomy of health sector managers and fate of resources 'released' through efficiency savings. In the second stage financial protection and equity in finance, health services quality, equity in health services utilization and in the distribution of resources, health system efficiency, transparency and accountability are assessed (24).

Chatham House developed a framework that concentrates on three areas: the domestic financing

of national health systems, the joint financing of global public goods for health, and the external financing of national health systems. To assess domestic financing of national health systems, it emphasizes on government's responsibilities in eight areas. 1) efficient, equitable and sustainable financing, 2) percent of gross domestic product (GDP) on health, 3) minimizing catastrophic and impoverishing out of pockets (OOPPs),4) improving revenue generation through mandatory and progressively prepaid pooled funds, 5) innovative taxation policies and tax compliance measures, 6) expand comprehensive primary health care for all population and set priorities to specialized care, 7) develop a systematic and multi criteria 
Y $\checkmark$ decision processes for priority-setting and 8) improve efficiency by focusing on corruption, r

¡vq The "aligning public financial management and health financing guide " is jointly prepared by

r^. Results for Development (R4D) and WHO's Department of Health Systems Governance and rA) Financing. This guide provides a framework for health and finance policy-makers for rur diagnosing misalignments and obstacles in regards to matching health financing objectives rAr and PFM systems and determining actions that can improve alignment. This guide is arranged $\lceil\wedge \varepsilon \quad$ into six assessment modules: laying the groundwork, health budget formulation, health budget rso execution and provider payment, budget accounting and reporting, fiscal sustainability, and rی५ options for achieving better alignment between PFM and health. Each module includes a set of rAv questions in summary tables outline the current situation and identify potential bottlenecks rMA (26).

r^৭ The "World Bank FinHealth PFM-in-health toolkit" identifies the main PFM-related rq. bottlenecks and opportunities to improving service delivery and make recommendations and (q) options for strengthening PFM arrangements. The framework provides a 'whole-of-system' rar view and a wide range of issues like supply-side service delivery issues, key PFM systems and ז ५ § and quantitatively. Undertaking a diagnostic using the toolkit will result in options for r9० strengthening PFM arrangements to support improved health service delivery (27).

\section{५ १ч Discussion}

५१^ In this paper, we reviewed Fifteen proposed health financing frameworks/ tools from 2001 to rq9 2020. These frameworks/ tools are developed attempting to assess and review health r.. financing. Since there are well-established and important contribution of "comparative 
r. $\quad$ perspective used in both academic field of public policy analysis and in more applied policy

$r \cdot r \quad$ studies" (28), frameworks/ tools help to assess health financing in different settings, the

r.r proposed table is helpful to identify promising framework and to enable categorizing and

$r \cdot \varepsilon \quad$ comparatively assessing frameworks.

r.० Despite being diverse, various health financing frameworks/ tools offer synergistic views to

$r .7$ the health financing system and provide a comprehensive picture of the health financing

$r \cdot v \quad$ system. They vary somehow in terms of the criteria and performance indicators, the position

$r \cdot \wedge$ of context and actors, and the degree to which they weight the importance of institutional and

$r \cdot q$ organizational factors. Across the majority of the mentioned frameworks/ tools, health

щ. financing was assessed alongside several dimensions, which has ten categories: a) contextual

M) factors, b) governance, c) social values, d) health information e) administrative capacity, f)

MIr laws and regulation, g) actors and stakeholders, h) goals and functions, I) incentives, j)

rir enforcement mechanisms.

r $\leqslant$ Nearly among analytical indicator-based frameworks reviewed, it seems there is a consistency

ro of their approach in identifying the performance indicators. The OASIS framework and

M14 "Health 2020" provide a supposedly well-defined categorization of performance indicators for

MIV revenue collection and pooling except strategic purchasing indicators, which is difficult to

Mis measure, assessed by data gathered through MOH policy documents and key informants

19 interviews. The unique value of these frameworks is that they provide a practical basis for in-

rr. depth analysis of structural, organizational and institutional components of health financing

rr) system, which bring them together within a wide perspective and link health financing

rr functions to the achievement of intermediate and broader health system objectives. Despite

r some similarities in their approach, there are still some differences in their stress on health

r $r \leqslant$ financing rules and organizational practice. However, the OASIS demonstrated less about

rro contextual factors as compared with the other mentioned frameworks $(8,11,22)$. Some 
rr countries like Korea, Cambodia and Vietnam, assessed their health financing system using

rrv OASIS framework, identified achievements and challenges and suggested policy options to

rrA maintain or accelerate progress towards universal coverage (29-31). Chua and his colleague in

rrq their study used the WHO Health Financing Strategy for the Asia Pacific Region (2010-2015)

rr. as the framework to evaluate the Malaysian healthcare financing system. This study shows

rr) that good governance would ensure adequate focus on the attainment of universal coverage

rre through allocating public spending on health (32).

There is a gradual shift towards a greater consideration of Public Finance Management. Kutzin

ש

rro emphasis on "Public Finance Management". Their framework included a descriptive approach

זr and detailed assessment of public sector financial management, relative power of different

rrv actors in the budget decision-making process, extent of decision-making autonomy of health

rru sector managers $(7,22)$.Describing health financing and PFM in a national health system, this

rrq tool offers analysis of the broader reform environment to inform moving to UHC.

rะ. The resource tracking and management framework provides a comprehensive data on

$r \varepsilon$ financial flows on the one hand and linkage between the financial statistics and the policy

r $\leqslant$ issues, analysis and interpretation on the other hand. Understanding this two-way relationship

$r \leqslant$ is critical for diagnosing misalignments between health financing policies and PFM good

$r \leqslant \varepsilon$ practices, the health financing system main obstacles to intervene, and actionable

$r \leqslant 0 \quad$ strategies for improving alignment $(20,26)$.

$r \leq \tau$ The FinHealth PFM-in-health toolkit aims to provide a 'whole-of-system' view, from the

$r \leqslant \vee$ upstream planning and budgeting to the downstream budget execution process grouped

$r \leqslant \wedge$ across 24 health functions, derived from a framework that brings together supply-side service

५ १ delivery issues, key PFM systems and health financing best practice. 
ro. Using this framework, the tool aims to make clear the linkages between identified PFM ro) problems, health financing in general, and service delivery. In this way, it is different to - for ror example - a PEFA for health approach, which focuses on the operation of PFM systems but ror with no specific focus as to how these affect service delivery.

ros The FinHealth toolkit does not set clear external benchmarks against which performance can roo be quantified and/or assessed. Instead, users of the tool work across questions grouped into ror 24 predefined health functions to draw their own narrative judgments based on the data rov collected, which are predominantly qualitative with some quantitative elements. Many of the ros questions within each identified function are in the form of 'To what extent...' and 'To what r०१ degree...', offering an open-ended response where users will have to determine for rч. themselves at what point a sufficient response has been provided. A few of the health ฯ function question areas are directly linked to PEFA indicators. It includes a number of rq questions and areas of focus that could be considered outside a strict definition of PFM r system functioning (e.g. payroll, procurement). The tool is certainly broad in that it puts rı forward a wide-ranging framework for understanding the interrelated issues of delivery rıo bottlenecks, PFM challenges and health financing best practice. A full response to all its q questions would provide a great deal of information on health service delivery and its PFM rฯ challenges. However, this also represents its major challenge: answering every question ฯ within the 24 health function areas - and collecting all the qualitative and quantitative data rч9 required to do it - may prove a significant logistical and resource challenge to carry out in its rr. $\quad$ entirety $(27)$

rv There are, on the other hand, some analytical frameworks like Chatham House, and rVr frameworks proposed by Kutzin, Mossialos and Meeseen that have devoted a deep attention to rvr contextual structure, institutional arrangements and valid information. Multi-faceted $r \vee \leqslant \quad$ interactions between these factors from the perspectives of the basic health financing functions 
rvo are critical. Absence of a set of comprehensive indicators for evaluating healthcare financing

r $\checkmark \uparrow$ in this group of frameworks is obvious $(9,10,16,25,33)$. The framework proposed by Hsiao

r $\checkmark v$ focuses more on health financing functions, their causal links and impact on different

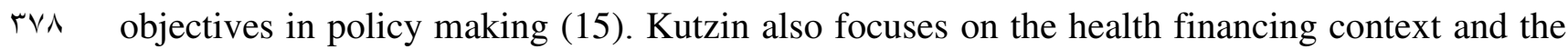

rvq interactions of policies. He conceptualizes the organizational and institutional arrangements of

r^. the components of health financing. He also stresses on the implementation of regulation and

rN information as key policy tools $(7,13,14)$. In the Gottret et al. study of Health Financing

rNT Revisited, common enabling factors across all or most of the nine studied countries were first

rлt identified. They include institutional and societal policy and implementation factors (7).

ґ^ะ Several studies are published with a focus on contextual factors. They showed that developing

rso health financing system within the particular macro-economic, socio-economic and political

rی context of the country and consideration of national context are of great importance (34-36).

rNV

r^^ Scope of frameworks/tools varies. Some scopes have a narrow perspective and focus only on

r^9 health financing and its functions while others assess the whole health system. The framework

rq. proposed by Hsiao and "Health 2020" aimed to analyze the health system as a whole. In their

rq) study of evaluation of Bulgaria's health financing system, Couffinhal et al. employed

rar WHO/World Bank taxonomy of health systems and the related goals and objectives. Their

rar assessment encompassed health service delivery, political economy and health financing. They

rqะ argued that the interaction of health infrastructure, human resources, information, drugs and

rq० medical supplies, technologies and stewardship together with health financing functions

r৭५ determined how well health system achieved its ultimate goals (37).

rav Ovretviet and Marmor believed that comparative health policy helped to "expand ґ १^ relationships among different countries by understanding their similarities and differences" r११ and by "adapting ideas that have worked elsewhere" (28). Quoting Rose and Marmor (1993, 
¿. 1997), it has been argued that "shaping of a common global framework for health financing is £. a fallacy in international health comparisons discussions as it overlooks the importance of $\varepsilon \cdot r \quad$ context" $(38)$

\&.r Health systems research focuses more on the "science of delivery" today, which means how

$\varepsilon \cdot \varepsilon \quad$ the guiding principles of the system are implemented in practice, in a given context. The issue «.० of what works and what does not, is increasingly based on the socioeconomic, political and ¿. 7 cultural context (37).Considering these variations which differ among countries, Mossialos $\varepsilon \cdot \vee$ emphasizes in his model on political considerations, economic activity, demographic profile, $\varepsilon \cdot \wedge$ environmental factors, external pressures and social values (10). Political and national «.9 traditions shape the values, which have complex interactions with institutions and «). organizational performance. Solidarity, cost-sharing arrangements and considerations of cost ¿। effectiveness all play an important part in the decision for the use of resources (39).

\&Ir Walt and Gilson considers essential role of general and health system context, content of \& policies and process in defining a country's vision, priorities, and course of action for ¿) $\leqslant$ improving health financing policies. Stakeholder's concerns, interests and positions are very §10 important in proposing health financing strategies. Involving stakeholders might eliminate \&17 societal resistance, increase society's support and decrease feasibility constraints (40, 41). ¿।V Zoidze et al. assess the impact of the "national program for Medical Assistance to the Poor" §\^ on equity and financial protection using the "policy triangle framework" (42). In one of the §19 studies looking at social health protection of the informal sector, the researchers focused on ¿r. the policy analysis approach developed by Walt and Gilson and considered context and the \&Y views of scholars, politicians, bureaucrats to investigate the main policy and operational EYr barriers (36). Meessen explicitly considered the magnitude of the "property rights" and Er defined the alignment of actor's incentives with principals' objectives and their behavioral $\varepsilon r \leqslant$ pattern (16). 
¿ro The World Bank framework provides eight areas for countries which try to establish a health ¿ry insurance system from the beginning or extend their current health insurance system. Bazyar ErV used this framework as a initial framework to study the challenges ahead of merging health ¿r^ insurance funds in Iran. Studying merger of the existing health insurance funds as a policy to ¿rq boost risk poolong is something new which has been emphasized in this framework. Much is ¿r. needed to be done to understand the experience, advantages, disadvantages, challenges, \&T facilitators, and process of merging health insurance funds in those countries which have sTr implemented this policy (43-45)

\& The evidence reviewed here shows that over time, more determinants of health financing as $\varepsilon r \varepsilon$ demographic, socioeconomic, environmental, institutional and political factors are considered ¿ro for assessment. However, none of the frameworks on its own covers all the elements. It is \& 4 necessary to develop a broad and coherence framework to ensure containing all of the factors \&TV affecting health financing to achieve UHC. Shaping of a comprehensive framework for health \&r^ financing has to contribute to identify effective policy interventions. Health financing analysis $\varepsilon r q$ is a laborious endeavor that usually needs diverse data and an expert team. It is sometimes «.. infeasible to access ready and user-friendly information, therefore it is important to apply to «s them with some flexibility.

\& $\quad$ It is worth clarifying that although our research methods are extensive, but there might be \& $\leqslant$ additional frameworks that we have not identified. For example, frameworks might have been $\varepsilon \leqslant \varepsilon$ remained as unpublished policy documents. There is not any rich literature implicating the «ะ० validity of these frameworks and the results of applying them in making policy decisions, so $\leq \leqslant 7$ more studies need to be carried out. Also development of a comprehensive framework was $\leqslant \leqslant \vee \quad$ beyond the scope of this paper. However, in the light of the importance of health financing «^^ and UHC, an examination of the published and either unpublished health financing conceptual 
«ะ१ frameworks is timely and fundamentally important and may give informative and timely clues

«o. to health policy makers.

$\{01$

sor

sor Conclusion

£० Given the complexity of health financing, there is no single, widely accepted framework for £०0 assessing financing of health sector. Health financing assessments need to cover a potentially ¿01 wide range of dimensions - depending on country context - and to take a holistic view of the sov financial system. There is an overlap in all frameworks as we expected and common elements s०^ are observed among current frameworks. There are also some dimensions that enough £०१ attention should be paid to, like political dimensions and the role of stakeholders. Some Łฺ. frameworks need a great and time consuming data gathering procedure. This comparative \&7) study suggests that simplifying concepts for policy makers should be a priority in the STr development of a coherent and comprehensive framework. Development such frameworks with explicit feasible recommendations, building on key links between contexts, power ¿ฯ relationships among system stakeholders, institutions and interactions between health ¿тo financing functions has a practical value. We hope that in seeking to build a practical ะ7ฯ framework, we at least help to give a comprehensive picture of health financing frameworks to ¿ TV initiate and facilitate more works as at present UHC \&SDG are in the global agenda.

\section{$\sum \uparrow$ Declarations:}

¿79 Ethics approval and consent to participate: The study received ethical approval from Tehran $\varepsilon \vee$ University of Medical Sciences.

\&v) Consent for publication: Not applicable.

¿Vr Availability of data and materials: Not applicable. 
¿vr Competing interests: Authors declare that they have no competing interests.

$\varepsilon \vee \varepsilon \quad$ Funding: There was no financial support for writing this article.

$\varepsilon \vee 0$

Authors' contributions: MAS and MB participated in designing the study, gathering data, and

$\varepsilon \vee T$

$\varepsilon \vee V$

$\varepsilon \vee \wedge$

$\varepsilon \vee 9$

$\varepsilon \wedge$. writing the manuscript. AR and $\mathrm{MB}$ made substantial contributions by reviewing and editing the manuscript and commenting and advising how to organize the manuscript. We confirm that the manuscript has been read and approved by all named authors and that there are no other persons who satisfied the criteria for authorship but are not listed. We further confirm that the order of authors listed in the manuscript has been approved by all of us.

Acknowledgements: This research is part of the thesis on "Policy analysis of universal health coverage financing in Iran "which was supported by Tehran University of Medical Sciences (TUMS).

\section{References}

1. Tangcharoensathien V, Mills A, Palu T. Accelerating health equity: the key role of universal health coverage in the Sustainable Development Goals. BMC medicine. 2015;13(1):101.

2. (WHO) WHO. Health systems: Improving performance. Geneva; 2000.

3. Evans DB, Etienne $C$. Health systems financing and the path to universal coverage. Bull World Health Organ. 2010;88(6):402.

4. Stuckler D, Feigl AB, Basu S, McKee M, editors. The political economy of universal health coverage. Background paper for the global symposium on health systems research Geneva: World Health Organization; 2010.

5. Ekman B. Community-based health insurance in low-income countries: a systematic review of the evidence. Health policy and planning. 2004;19(5):249-70.

6. Ahmed S, Annear PL, Phonvisay B, Phommavong C, Cruz VdO, Hammerich A, et al. Institutional design and organizational practice for universal coverage in lesser-developed countries: Challenges facing the Lao PDR. Social Science \& Medicine. 2013;96:250-7.

7. Gottret PE, Schieber G, Waters H. Good practices in health financing: lessons from reforms in low and middle-income countries: World Bank Publications; 2008.

8. Di McIntyre, Kutzin J. Guidance on conducting a situation analysis of health financing for universal health coverage. WHO; 2014. Contract No.: Version 1.0

9. Kutzin J. Health financing policy: a guide for decision-makers. Health financing policy paper Copenhagen, WHO Regional Office for Europe. 2008;24.

10. Mossialos E, Dixon A, Figueras J, Kutzin J. Funding health care: options for Europe. Open University Press Maidenhead; 2002. 
11. Mathauer laGC. The role of institution design and organizational practice for health financing

0.1 performance and universal coverage. Health Policy. 2011; 99:183-92.

0.9

12. Antunes A, Saksena P. Health financing system review 2008: Options for universal coverage. Geneva: Ministry of Health Rwanda and World Health Organization. 2009.

13. Kutzin J, Cashin C, Jakab M. Implementing health financing reform. World Health Organisation on behalf of the European Observatory on Health Systems and Policies. 2010;7(5). 14. Kutzin J. A descriptive framework for country-level analysis of health care financing arrangements. Health policy 2001;56(3):171-204.

15. Hort K, Goss J, Hopkins S, Annear P. Conceptual frameworks, health financing data and assessing performance: A stock-take of tools for health financing analysis in the Asia-Pacific region. 2010 .

16. Bertone MP, Meessen B. Studying the link between institutions and health system performance: a framework and an illustration with the analysis of two performance-based financing schemes in Burundi. Health policy and planning. 2012:czs124.

17. WHO. Health financing strategy for the Asia Pacific region 2010-2015.

. Geneva: World Health Organization; 20092009.

18. Wang H, Switlick K, Ortiz C, Zurita B, Connor C. Health insurance handbook: how to make it work: The World Bank; 2011.

19. Bazyar M, Rashidian A, Sakha MA, Mahdavi MRV, Doshmangir L. Combining health insurance funds in a fragmented context: what kind of challenges should be considered? BMC Health Services Research. 2020;20(1):1-14.

20. Wang H, Kress DH, Berman P. From Resources to Beneficiaries: Introducing an End-to-End Resource Tracking and Management Framework and its Application for Primary Health Care Development in LMICs. World Scientific Book Chapters. 2020:3-21.

21. OECD/Eurostat/World Health Organization (2017). "Classification of Health Care Financing Schemes (ICHAHF)", in A System of Health Accounts 2011: Revised edition, OECD Publishing, Paris.

22. Islam M. Health systems assessment approach: a how-to manual. Health systems assessment approach: a how-to manual. 2007.

23. Todini N. Guided Self-Assessment of Public Financial Management Performance (PFMP-SA) A Toolkit for Health Sector Managers. Bethesda, MD: Health Finance \& Governance Project, Abt Associates Inc. December 2013.

24. Kutzin J, Witter S, Jowett M, Bayarsaikhan D, Organization WH. Developing a national health financing strategy: a reference guide: World Health Organization; 2017.

25. Rottingen J, Ottersen T, Ablo A, Arhin-Tenkorang D, Benn C, Elovainio R, et al. Shared responsibilities for health: a coherent global framework for health financing; final report of the Centre on Global Health Security Working Group on Health Financing: The Royal Institute of International Affairs; 2014.

26. Cashin C, Bloom D, Sparkes S, Barroy H, Kutzin J, O'Dougherty S, et al. Aligning public financial management and health financing: sustaining progress toward universal health coverage: World Health Organization; 2017.

27. Chukwuma A, Gurazada S, Jain M, Tsaturyan S, Khcheyan M. FinHealth Armenia: Reforming Public Financial Management to Improve Health Service Delivery. World Bank; 2020.

28. Blank RH, Burau V. Comparative health policy: Palgrave Macmillan; 2013.

29. Mathauer I, Xu K, Carrin G, Evans DB. An analysis of the health financing system of the Republic of Korea and options to strengthen health financing performance. Geneva, WHO. 2009. 30. Tien TV PH, Mathauer I, Phuong NTK. A health financing review of Viet Nam with a focus on social health insurance. Geneva: : World Health Organization. ; 2011.

31. AF A. . Summary Report The Health Financing System Assessment In Cambodia. . 2009. 32. Chua HT, Cheah JCH. Financing Universal Coverage in Malaysia: a case study. BMC Public Health. 2012;12(1):S7. 
33. Bank AD. Creating financing opportunities for universal health coverage in Africa: From Aspiration to Action. LUSAKA, ZAMBIA; 2013.

34. Maeda A, Edson Araujo, Cheryl Cashin, Joseph Harris,, Naoki Ikegami aMRR. Universal Health Coverage for Inclusive and Sustainable Development: A Synthesis of 11 Country Case Studies: Directions in Development. Washington, DC:

World Bank. doi:10.1596/978-1-4648-0297-3. License: Creative Commons Attribution CC BY 3.0 IGO; 2014.

35. Vargas JR, Muiser J. Promoting universal financial protection: a policy analysis of universal health coverage in Costa Rica (1940-2000). Health Research Policy and Systems. 2013;11.

36. Annear PL, Ahmed S, Ros CE, Ir P. Strengthening institutional and organizational capacity for social health protection of the informal sector in lesser-developed countries: A study of policy barriers and opportunities in Cambodia. Social Science \& Medicine. 2013;96:223-31.

37. Couffinhal AN, Ha Thi Hong; Haazen, Dominic S.; Holla, Alaka; Hendriks, Ronald Hendrikus Maria; Lavado, Rouselle F.; Lopert, Ruth; Postolovska, Iryna; Schieber, George; Shah, Jugna Jiten; Stoynova, Sylvia Nikolova; Streveler, Dennis J.; Francis, Gabriel C.;. Bulgaria - Health financing RAS : final report on health financing diagnostic and review of envisaged reforms. 2015. Contract No.: ACS15242.

38. McPake B, Mills A. What can we learn from international comparisons of health systems and health system reform? Bulletin of the World Health Organization. 2000;78(6):811-20.

39. Littlejohns P, Weale A, Chalkidou K, Teerwattananon Y, Faden R, Clark S, et al. Social values in health priority setting: a conceptual framework. Journal of health organization and management. 2012;26(3):293-316.

40. Walt G, Gilson L. Reforming the health sector in developing countries: the central role of policy analysis. Health policy and planning. 1994;9(4):353-70.

41. Roberts MH, W.; Berman, P.; Reich, M. Getting Health Reform Right: A Guide to Improving Performance and Equity. New York, United States: Oxford University Press; 2003.

42. Zoidze A, Rukhadze N, Chkhatarashvili K, Gotsadze G. Health Insurance for Poor-Georgia's Path to Universal Coverage. Working Paper: Curatio International Foundation, viewed 1 February 2014, http://www. curatiofoundation. org; 2012.

43. Bazyar M, Yazdi-Feyzabadi V, Rahimi N, Rashidian A. What are the potential advantages and disadvantages of merging health insurance funds? A qualitative policy analysis from Iran. BMC public health. 2020;20(1):1-15.

44. Bazyar M, Rashidian A, Sakha MA, Doshmangir L, Rahimi N, Ranjbar M, et al. Stakeholders analysis of merging social health insurance funds in Iran: what kind of interests they may gain or lose? The International journal of health planning and management. 2019;34(1):157-76. 45. Bazyar M, Rashidian A, Kane S, Mahdavi MRV, Sari AA, Doshmangir L. Policy options to reduce fragmentation in the pooling of health insurance funds in Iran. International journal of health policy and management. 2016;5(4):253. 


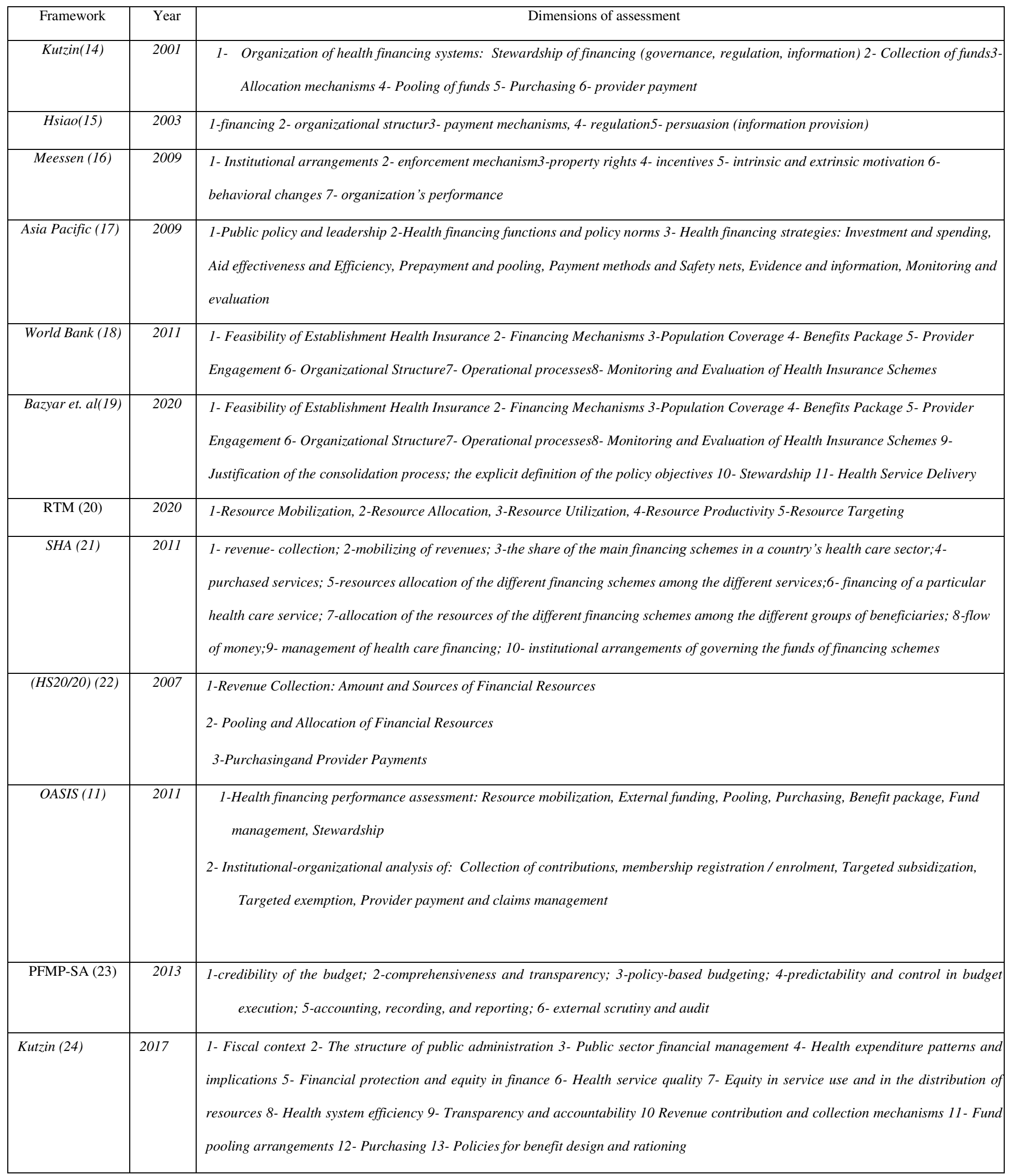




\begin{tabular}{|c|c|c|}
\hline $\begin{array}{l}\text { Chatham House } \\
\text { (25) }\end{array}$ & 2014 & $\begin{array}{l}\text { 1- Domestic financing of national health systems } \\
\text { 2- the joint financing of global public goods for health } \\
\text { 3- the external financing of national health systems }\end{array}$ \\
\hline Cashin (26) & 2017 & $\begin{array}{l}\text { 1-laying the groundwork, 2-health budget formulation, 3-health budget execution and provider payment, 4-budget accounting and } \\
\text { reporting,5-fiscal sustainability, and 6-options for achieving better alignment between PFM and health. }\end{array}$ \\
\hline$P F M(27)$ & 2020 & $\begin{array}{l}\text { 1-wide range of issues like supply-side service delivery issues, key PFM systems and health financing best practice grouped across } 24 \\
\text { health functions }\end{array}$ \\
\hline
\end{tabular}

$7 . r$

$7 \cdot r$

7.5

7.0

7.7

$7 . V$

7.1

7.9

7).

71)

7)

TIT

7)

710 